\title{
How do we perform hyperthermic intraperitoneal chemotherapy in ovarian cancer? - a narrative review
}

\author{
Hyeong In $\mathrm{Ha}^{1}$, Myong Cheol $\mathrm{Lim}^{2}$
}

${ }^{1}$ Department of Obstetrics and Gynecology, Pusan National University Yangsan Hospital, Pusan National University School of Medicine, Yangsan, Korea; ${ }^{2}$ Research Institute and Hospital, National Cancer Center, Goyang, Korea

Contributions: (I) Conception and design: All authors; (II) Administrative support: All authors; (III) Provision of study materials or patients: All authors; (IV) Collection and assembly of data: HI Ha; (V) Data analysis and interpretation: HI Ha; (VI) Manuscript writing: HI Ha; (VII) Final approval of manuscript: All authors.

Correspondence to: Myong Cheol Lim, MD, PhD. Center for Gynecologic Cancer, Research Institute and Hospital, National Cancer Center, 323 , Ilsan-ro, Ilsandong-gu, Goyang-si, Gyeonggi-do 10408, Korea. Email: gynlim@gmail.com.

\begin{abstract}
Ovarian malignancy is a leading cause of death caused by gynecologic cancer worldwide because it is mainly found in the advanced stage and recurs in most patients even after cytoreductive surgery and intravenous (IV) chemotherapy. Prevention of recurrence of primary disease and treatment of recurrent ovarian cancer are still remained as major interest and lots of researchers investigate novel treatment to find optimal method. Even though intraperitoneal (IP) chemotherapy turns out to increase the overall survival, it is not widely used because of adverse event. As an alternative treatment for IP chemotherapy, hyperthermic intraperitoneal chemotherapy (HIPEC) is emerging a new way. Thanks to much research and use in other cancer species, such as the colorectal cancer cytoreductive surgery followed by HIPEC is becoming a promising treatment. However, randomized controlled trials and unbiased data in ovarian cancer patients are still needed for the establishment of therapy. Moreover, among the current situation in which treatments such as bevacizumab or PARP inhibitor have been found to be effective and have been widely used, it may be necessary to establish the role in the combination of HIPEC. This article is a comprehensive review of the HIPEC in ovarian cancer to introduce techniques, treatment results, and clinical trials of HIPEC.
\end{abstract}

Keywords: Hyperthermic intraperitoneal chemotherapy (HIPEC); ovarian cancer; surgery

Submitted Mar 30, 2020. Accepted for publication Mar 08, 2021.

doi: $10.21037 /$ gs-20-425

View this article at: http://dx.doi.org/10.21037/gs-20-425

\section{Introduction}

Substantial number of ovarian cancer patients is typically diagnosed at an advanced age. Thus far, there is lack of methods recommended for screening and early diagnostics of this disease (1). The standard treatment for advanced ovarian cancer involves cytoreduction surgery followed by six cycles of intravenous (IV) chemotherapy with carboplatin and paclitaxel or three cycles of neoadjuvant chemotherapy and interval cytoreductive surgery (2). In spite of standard treatment, appropriate treatments of advanced or recurrent ovarian malignancy still remains an important question. Although $70 \%$ to $80 \%$ of patients respond to initial therapy, typically, only $15 \%$ are cured, with the remaining developing drug-resistant recurrent disease (3). Considering the high and fairly constant rate of recurrent disease in epithelial ovarian cancer women, identifying the best treatment of recurrence is one of the main objectives for the oncologists, to prolong the time to secondary progression and finally extending overall survival (4).

Hyperthermic intraperitoneal chemotherapy (HIPEC), is a unique hyperthermia therapy used after debulking surgery for the management of advanced abdominal cancers such as pseudomyxoma peritonei or malignant peritoneal mesothelioma. Several articles report favorable result about 
HIPEC in ovarian cancer-Such method is a very appealing therapeutic option for advanced or recurrent ovarian cancer because of the previously reported theoretical rationale for tumors affecting the peritoneum $(5,6)$.

The peritoneal cavity is the major site of disease in ovarian malignancy, and the bulk of the tumor is located within the peritoneum (7). Therefore, there were lots of effort to try intraperitoneal (IP) chemotherapy as a reasonable attempt. The Gynecologic Oncology Group conducted a randomized, phase III trial that compared IV paclitaxel plus cisplatin with IV paclitaxel plus IP cisplatin and paclitaxel in patients with stage III ovarian cancer. In that study, the overall survival of IV plus IP chemotherapy group was extended (8). Although IP chemotherapy prolonged the overall survival, it was not widely used due to more frequent and severe adverse effects than IV chemotherapy.

HIPEC is an intraoperative chemotherapy as onetime treatment under general anesthesia at the time of debulking surgery. Therefore, concerns about poor compliance of patients can be reduced. Although exact cytotoxic mechanisms associated with hyperthermic temperatures are unclear, there can be several advantages to adopt HIPEC as compared with IP chemotherapy. Hyperthermia has been shown to augment the cytotoxic effect of some chemotherapeutic agents by increasing DNA-crosslinking and increasing tumor penetration (9). In addition, by giving the cytotoxic agent intraoperatively, drug exposure is optimal secondary to direct contact with the remaining microscopic cancer cells without the barriers of postoperative adhesions and can be guaranteed optimal distribution of the chemotherapy. In this article, we examine the surgical method of HIPEC and we present the article in accordance with the Narrative review reporting checklist (available at http://dx.doi.org/10.21037/gs-20-425).

\section{Techniques for HIPEC}

The whole procedure of HIPEC can be divided into exploration, debulking, and chemoperfusion. Before the full-scale operation, the surgeons open the abdomen and evaluate the peritoneal cancer. Prior to HIPEC, the abdomen and pelvis are to be cleared of all visible tumor masses and peritoneal nodules. Only structures covered by disease are resected. Opinions supported by all data shows that the more cytoreduction is completed, the greater the benefits that will occur from HIPEC treatment. Not only complete surgical cytoreduction, Sugarbaker et al. described various variables to consider for the successful implementation of HIPEC (10). Proper selection of chemotherapy agents, proper duration time of HIPEC, and appropriate temperature for peritoneal metastases were important. Figure 1 schematically shows the circuit of HIPEC

Several different methodologies for HIPEC exist and can be classified into diverse categories, according to the researcher's preference. In this article, the perfusion technique for HIPEC can be roughly classified into two categories: open-abdomen technique, closed-abdomen technique. Each HIPEC method has its own advantages and disadvantages. In addition, a few special methods are available such as laparoscopic HIPEC, depending on the situation.

\section{HIPEC regimens in ovarian cancer}

Usually, platinum-based agents (cisplatin, carboplatin, or oxaliplatin) are employed for HIPEC, and used as a single agent or in combination. To the exclusion of extreme value, 75 to $100 \mathrm{mg} / \mathrm{m}^{2}$ dose of cisplatin, 50 to $60 \mathrm{mg} / \mathrm{m}^{2}$ dose of paclitaxel are widely used. In the case of platinum resistant recurred ovarian cancer, doxorubicin and mitomycin $\mathrm{C}$ are also used. To treat a platinum resistant subgroup, Spiliotis et al. used a combination of doxorubicin, paclitaxel, and mitomycin (11). The temperature of perfusate ranges from 40 to $46{ }^{\circ} \mathrm{C}$. However, the majority are from 41 to $43{ }^{\circ} \mathrm{C}$ (4,11-22) and the duration of procedure ranges from 30 to 120 minutes in accordance with operator's directions (23-35). Even though optimum temperature has not been determined, chemotherapeutic agents applied for HIPEC are usually chemically stable.

\section{Open-abdomen technique}

HIPEC can be performed in various ways and in fact, it is implemented slightly differently depending on the preferences and know-how of the surgeon. However, there is no clear evidence that a particular method is superior than the others. The open method is usually performed using the "Coliseum technique" described by Sugarbaker $(10,36)$.

At the end of cytoreductive phase, a frame that can be installed $15-20 \mathrm{~cm}$ above the abdominal surface of a lying patient is required. Lift the skin at the edge of abdominal incision and spread it by hanging it on the aforementioned retractor. By lifting all the periphery as above, the surgeon made the abdominal cavity one huge pocket, so it can be 


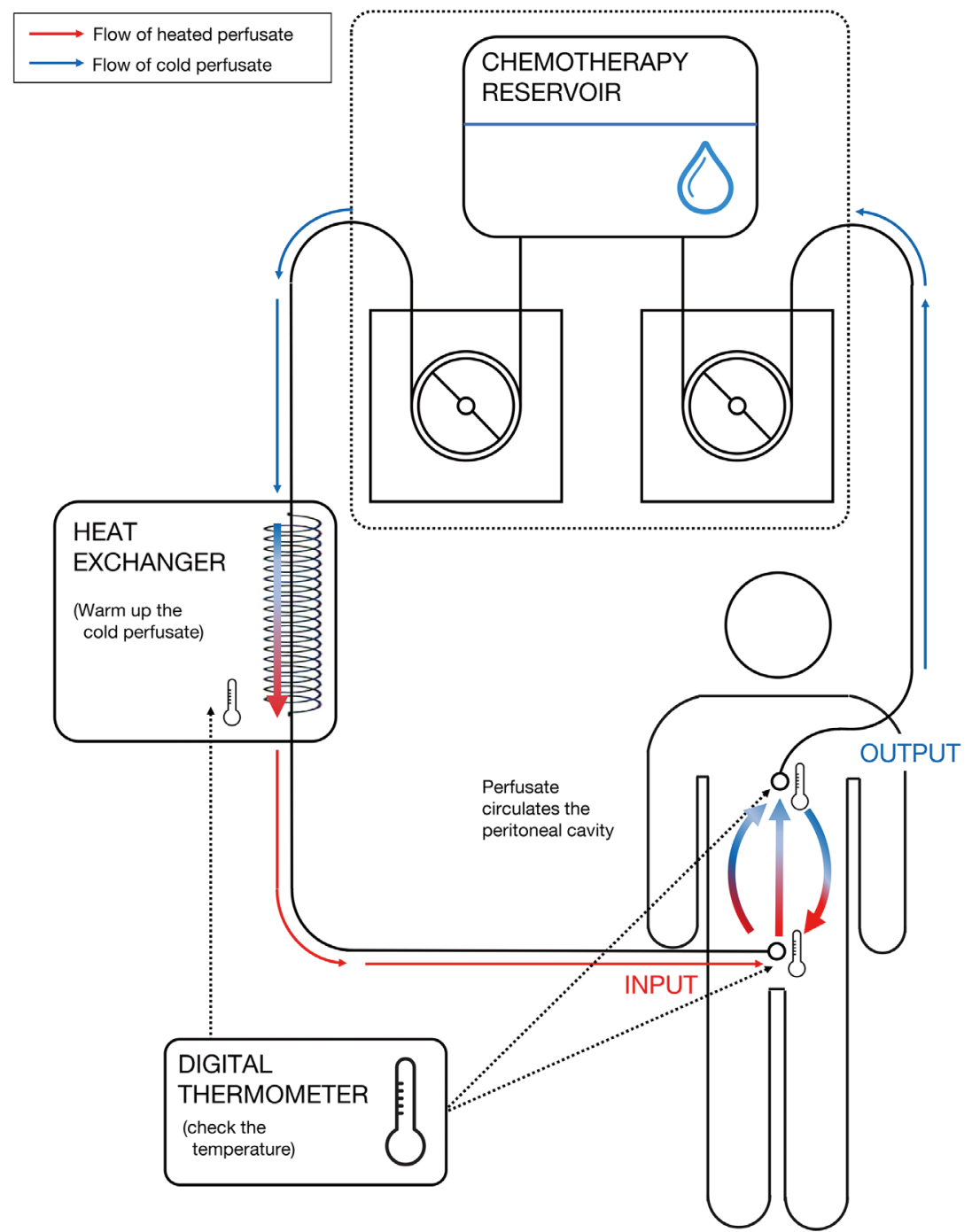

Figure 1 Schematic drawing — circuit of HIPEC. HIPEC, hyperthermic intraperitoneal chemotherapy.

a temporary reservoir for chemotherapy solution. Two to four liters of perfusate can be contained in the abdominal and pelvic cavity. Sugarbaker et al. used a single inflow (Tenckhoff catheter) and four outflow tubes (closed suction drains) placed through the lateral aspect of the abdominal wall at the end of cytoreductive phase for hyperthermic perfusate circulation (36). The inflow catheter is placed beneath the right hemidiaphragm. This inflow catheter number, outflow tube number, and the location of catheters can be changed according to the surgeon's preferences and needs. The important element is that chemotherapeutic agent flows through the whole abdominal cavity, without deviation. Temperature probes are used to monitor IP temperature. The number of temperature probes used during HIPEC may vary, though one probe should measure the temperature of the inflow and the other should be at a distance from the pelvic cavity. However, if more intensive and critical temperature monitoring is needed, the number of probes can be added. Probe tips may be secured to the tip of the matched drains to prevent deviation.

An impermeable disposable silastic sheet conceals the entire operative field in order to stop the chemotherapy solution from leakage. According to the open technique, a plastic sheet covered abdomen and the operator can access the abdominal cavity by making a cruciate incision in the middle of the sheet. The surgeon can operate the 
abdominal organs by inserting his hand into the abdominal cavity through the incision of the sheet, and can prevent uneven delivery of heated anticancer drugs. At this time, the surgeon should take full care of the operator's own safety, such as wearing two layers of gloves. A smoke evacuator can be used to protect the surgical-staff from aerosolized chemotherapeutic agent vaporized during the HIPEC. A roller pump pushes the perfusate into the abdominal cavity through the Tenckhoff catheter and drains it through the exit lines, with a flow rate $\sim 1 \mathrm{~L} / \mathrm{min}$. A heat exchanger keeps the temperature of infused fluid at $43-45^{\circ} \mathrm{C}$, therefore that of IP fluid is maintained at $41-43^{\circ} \mathrm{C}(37)$. The perfusate is reheated adequate hyperthermic temperature when it circulated between the heat exchanger and the reservoir. The anticancer drug is then mixed to the established circuit, and then measure the perfusion time.

Elias et al. reported that the use of the coliseum technique is the best technique from the point of thermal homogeneity and spatial diffusion (38). The open technique and the closed technique, each has its advantages. homogeneous exposition of peritoneal surfaces to the therapy can be allowed because the intra-abdominal viscera may be manipulated during perfusion. In addition, excessive heating of peritoneal cavity was decreased theoretically. However, open technique takes longer time to achieve target temperature due to heat dissipation. Moreover, it is difficult to ensure that the perfusate evenly reaches the anterior abdominal wall because of the risk of overflow.

\section{Closed-abdomen technique}

In this case, the circulation of perfusate occurs after the abdomen is closed. Two outflow tubes (one with a temperature sensor attached) are placed on deep pelvis and connected by another $\mathrm{Y}$ connector to a single outflow tube. Two inflow tubes (one with a temperature sensor attached at the tip) are connected by a $\mathrm{Y}$ connector to a single inflow tube and placed above the right lobe of the liver and in left upper quadrant of abdomen. The outflow tubes are laid behind the small bowel at the back of abdomen and brought out in the upper part of the incision, with the inflow tubing coming out of the lower part of the incision (39). When the abdomen is closed, some surgeons close the skin (not the rectus fascia) only and re-open the abdomen after HIPEC procedure is ended. In this method, after hyperthermic perfusion, the abdomen is reopened and anastomoses, stoma, and drain placement are performed. The abdomen is then closed in a standard order. On the other hand, some surgeons prefer to close completely fascia and skin. Anastomoses and stoma are performed before abdominal wall closure. This way, there is no need to reopen the abdomen at the end of HIPEC, and catheters used for perfusion are used as drains during postoperative care (40). After the abdomen is sealed, the antitumor agent in perfusate is circulated in limited volume of abdominal cavity. A larger volume of perfusate is generally needed to set closed circuit compared with the open technique, and the higher abdominal pressure can be obtained during perfusion that may promote drug penetration into tissue (41).

\section{Comparisons between open- and closed-abdomen technique}

Both open and closed abdomen HIPEC method have their own strengths and weaknesses. The open technique facilitates even distribution of heat and chemotherapy agent, with the disadvantage of unwilled heat dissipation and leakage of cytotoxic agents. On the other hand, the closed technique avoids heat loss and drug leakage, assist drug penetration. However, the technique does not guarantee homogeneous circulation of the perfusate (Table 1) and this may cause increased systemic uptake and focal hyperthermic injury, which may immediate postoperative ileus, fistula, and bowel perforation (42).

In spite of the lots of studies and reviews reported on HIPEC in the last few years, there is limited report from randomized prospective trials to definitively determine which method has better outcomes for patients. A PubMed search using the search terms "HIPEC" and "technique" yields 174 results. After the exclusion of the study about clinical outcomes of HIPEC, we were able to have 31 studies about surgical techniques of HIPEC. A single arm pioneer report on experimental surgical techniques was also excluded. Excluding questionnaire survey, review article, studies on anesthesia during HIPEC treatment and about the management of surgical patients, there were 4 comparative studies between open technique and closed technique for the treatment of peritoneal carcinomatosis. Finally, after subtracting two animal studies, there were only two retrospective comparative studies for the comparisons of open and closed techniques. Both studies conclude that there is no difference between open and closed technique $(43,44)$ (Table 2).

It is recommended to select the possible surgical method at that time according to the preference of the operator, more research is needed to accurately know the superiority. 
Table 1 Comparison of open and closed methods of HIPEC

\begin{tabular}{lcc}
\hline Points to compare & Open & Closed \\
\hline Heat distribution & More uniform & Less uniform \\
Chemotherapy distribution & More uniform & Less uniform \\
Heat dissipation & More & Less \\
Time to achieve target temperature & Longer & Shorter \\
Chemotherapy exposure & Increased & Minimized \\
Thermal injury risk of patients & Can be minimized & Possible \\
\hline
\end{tabular}

HIPEC, hyperthermic intraperitoneal chemotherapy.

Table 2 Comparison of clinical outcomes of open and closed technique of HIPEC

\begin{tabular}{|c|c|c|c|c|c|c|c|}
\hline Author & Cases [n] & Disease [n] & Regimen & Time & Temperature & Main endpoints & Result \\
\hline
\end{tabular}

HIPEC, hyperthermic intraperitoneal chemotherapy; NSS, no statistical significance; CVP, central venous pressure; HR, heart rate; SBP, systolic blood pressure.

\section{Other HIPEC methods}

There is a variation of the open technique that uses a device called "peritoneal cavity expander" (PCE) and this method used and described mainly in Japan $(45,46)$. The use of the PCE is very rare (if any) at the present time (40).

Lotti et al. reported a novel method, the laparoscopyenhanced HIPEC technique (47) to combine the advantages of the two techniques. Ports can be located through trocar sites under laparoscopic guidance. They performed cytoreductive surgery first through a conventional midline incision from the xiphoid process to the symphysis pubis first. After the end of cytoreductive surgery, they inserted the four Jackson-Pratt drains in the abdominal cavity. After the skin closure, three $12-\mathrm{mm}$ balloon trocars are placed in the quartile point of incision. Heated $\mathrm{CO}_{2}$ is then insufflated through the trocha and they stirred up the intra-abdominal contents during the HIPEC under the direct vision.

\section{Surgical-staff exposure of chemotherapeutic agent during HIPEC}

The effects of repeated, prolonged occupational inhalation and contact to chemotherapeutic agents remain unknown. However, even if the risk has not been proven, it is clear that best efforts should be made to protect surgical-staff from potential risks. Theoretically, harmful side effects (gastrointestinal toxicity, bone marrow toxicity, hair loss, and so on) may occur by absorption of low dose of such chemotherapy agent and repeated exposure (40). In addition, relatively long time for operation (1012 hours) and using high-voltage devices can result in not only chemotherapeutic agent inhalation, but also surgical smoke. For the safe HIPEC procedure, standard protective guidelines should be provided and obeyed.

Direct contact with mucous membranes or skin can cause mucositis or dermatitis. Moreover, inhalation of 
vaporized agent particles can cause systemic problem. Even if it doesn't exceed the boiling point, cytotoxic drugs can be vaporized due to the hyperthermia.

Several studies have addressed the safety of open techniques. Samples of blood and urine were collected from surgical-staff during oxaliplatin-based HIPEC treatment with coliseum technique HIPEC. As a result, no detectable signs of platinum in the blood or urine of either the surgeon, during six 30-minute HIPEC perfusions of oxaliplatin were detected (48). Mitomycin C level in the urine of members of the HIPEC team was evaluated in other study and no potential risk of exposure was found with efforts to safety standards $(49,50)$. However, the studies mentioned above are not studies of cisplatin, which is often used to HIPEC of ovarian cancer. Furthermore, lung fibrosis, bone marrow suppression, and other longterm effects cannot be evaluated. Therefore, we need to plan the long-term investigation for delayed adverse effects of HIPEC to surgical staff.

\section{Conclusions}

Although lots of studies have shown the effectiveness of HIPEC, some questions were still remained. Many recent studies are showing the improved impact of Cytoreductive surgery and HIPEC on clinical outcomes in the ovarian cancer patients. However, in order to apply this to real world, proper and thorough surgery will be necessary. Whether open or closed technique, surgery will be performed according to the judgment of the surgeon, but I look forward to further development of the surgical method.

Other problem is that which is the best chemotherapy agent as a perfusate. Generally platinum based chemotherapy agent such as cisplatin are used, and even used when performing HIPEC in platinum resistant patients. However, it is not clear which is most effective. In addition, it is unclear which is the optimal temperature for HIPEC. Ongoing and newly planned clinical trials will be able to answer the questions. Although minor variations for implementation of HIPEC, its adoption into routine clinical practice is expected to happen in the near future.

\section{Acknowledgments}

Funding: None.

\section{Footnote}

Provenance and Peer Review: This article was commissioned by the Guest Editors (Sang Yoon Park, Jae Weon Kim) for the series "Ultra-Radical Surgery in Ovarian Cancer: Surgical Techniques for Gynecologic Oncologist" published in Gland Surgery. The article was sent for external peer review organized by the Guest Editors and the editorial office.

Reporting Checklist: The authors have completed the Narrative review reporting checklist. Available at http:// dx.doi.org/10.21037/gs-20-425

Conflicts of Interest: Both authors have completed the ICMJE uniform disclosure form (available at http:// dx.doi.org/10.21037/gs-20-425). The series "UltraRadical Surgery in Ovarian Cancer: Surgical Techniques for Gynecologic Oncologist" was commissioned by the editorial office without any funding or sponsorship. Both authors have no other conflicts of interest to declare.

Ethical Statement: The authors are accountable for all aspects of the work in ensuring that questions related to the accuracy or integrity of any part of the work are appropriately investigated and resolved.

Open Access Statement: This is an Open Access article distributed in accordance with the Creative Commons Attribution-NonCommercial-NoDerivs 4.0 International License (CC BY-NC-ND 4.0), which permits the noncommercial replication and distribution of the article with the strict proviso that no changes or edits are made and the original work is properly cited (including links to both the formal publication through the relevant DOI and the license). See: https://creativecommons.org/licenses/by-nc-nd/4.0/.

\section{References}

1. Cortez AJ, Tudrej P, Kujawa KA, et al. Advances in ovarian cancer therapy. Cancer Chemother Pharmacol 2018;81:17-38.

2. van der Burg ME, Van Lent M, Buyse M, et al. The effect of debulking surgery after induction chemotherapy on the prognosis in advanced epithelial ovarian cancer. $\mathrm{N} \mathrm{Engl} \mathrm{J}$ Med 1995;332:629-34. 
3. Armstrong DK. Relapsed ovarian cancer: challenges and management strategies for a chronic disease. Oncologist 2002;7:20-8.

4. Fagotti A, Costantini B, Petrillo M, et al. Cytoreductive surgery plus HIPEC in platinum-sensitive recurrent ovarian cancer patients: a case-control study on survival in patients with two year follow-up. Gynecol Oncol 2012;127:502-5.

5. Sugarbaker PH, Van der Speeten K, Stuart OA. Pharmacologic rationale for treatments of peritoneal surface malignancy from colorectal cancer. World J Gastrointest Oncol 2010;2:19.

6. Sticca RP, Dach BW. Rationale for hyperthermia with intraoperative intraperitoneal chemotherapy agents. Surg Oncol Clin N Am 2003;12:689-701.

7. Cowan RA, O'Cearbhaill RE, Zivanovic O, et al. Current status and future prospects of hyperthermic intraoperative intraperitoneal chemotherapy (HIPEC) clinical trials in ovarian cancer. Int J Hyperthermia 2017;33:548-53.

8. Armstrong DK, Bundy B, Wenzel L, et al. Intraperitoneal cisplatin and paclitaxel in ovarian cancer. N Engl J Med 2006;354:34-43.

9. Los G, Van Vugt MJ, Den Engelse L, et al. Effects of temperature on the interaction of cisplatin and carboplatin with cellular DNA. Biochem Pharmacol 1993;46:1229-37.

10. Sugarbaker PH, Van der Speeten K. Surgical technology and pharmacology of hyperthermic perioperative chemotherapy. J Gastrointest Oncol 2016;7:29.

11. Spiliotis J, Halkia E, Lianos E, et al. Cytoreductive surgery and HIPEC in recurrent epithelial ovarian cancer: a prospective randomized phase III study. Ann Surg Oncol 2015;22:1570-5.

12. Delotte J, Arias T, Guerin O, et al. Hyperthermic intraperitoneal chemotherapy for the treatment of recurrent ovarian cancer in elderly women. Acta Obstet Gynecol Scand 2015;94:435-9.

13. Cascales-Campos PA, Gil J, Feliciangeli E, et al. The role of hyperthermic intraperitoneal chemotherapy using paclitaxel in platinum-sensitive recurrent epithelial ovarian cancer patients with microscopic residual disease after cytoreduction. Ann Surg Oncol 2015;22:987-93.

14. Königsrainer I, Horvath P, Struller F, et al. Cytoreductive surgery and hyperthermic intraperitoneal chemotherapy in recurrent epithelial ovarian cancer with peritoneal metastases: a single centre experience. Langenbecks Arch Surg 2014;399:589-94.

15. Zivanovic O, Abramian A, Kullmann M, et al. HIPEC ROC I: A phase i study of cisplatin administered as hyperthermic intraoperative intraperitoneal chemoperfusion followed by postoperative intravenous platinum-based chemotherapy in patients with platinumsensitive recurrent epithelial ovarian cancer. Int J Cancer 2015;136:699-708.

16. Bakrin N, Bereder JM, Decullier E, et al. Peritoneal carcinomatosis treated with cytoreductive surgery and Hyperthermic Intraperitoneal Chemotherapy (HIPEC) for advanced ovarian carcinoma: a French multicentre retrospective cohort study of 566 patients. Eur J Surg Oncol 2013;39:1435-43.

17. Argenta PA, Sueblinvong T, Geller MA, et al. Hyperthermic intraperitoneal chemotherapy with carboplatin for optimally-cytoreduced, recurrent, platinum-sensitive ovarian carcinoma: a pilot study. Gynecol Oncol 2013;129:81-5.

18. Gouy S, Uzan C, Pautier P, et al. Results of oxaliplatinbased hyperthermic intraperitoneal chemotherapy in recurrent ovarian granulosa cell tumors. Eur J Obstet Gynecol Reprod Biol 2013;170:464-7.

19. Deraco M, Virzì S, Iusco DR, et al. Secondary cytoreductive surgery and hyperthermic intraperitoneal chemotherapy for recurrent epithelial ovarian cancer: a multi-institutional study. BJOG 2012;119:800-9.

20. Ceelen WP, Van Nieuwenhove Y, Van Belle S, et al. Cytoreduction and hyperthermic intraperitoneal chemoperfusion in women with heavily pretreated recurrent ovarian cancer. Ann Surg Oncol 2012;19:2352-9.

21. Roviello F, Pinto E, Corso G, et al. Safety and potential benefit of hyperthermic intraperitoneal chemotherapy (HIPEC) in peritoneal carcinomatosis from primary or recurrent ovarian cancer. J Surg Oncol 2010;102:663-70.

22. Muñoz-Casares FC, Rufián S, Rubio MJ, et al. The role of hyperthermic intraoperative intraperitoneal chemotherapy (HIPEC) in the treatment of peritoneal carcinomatosis in recurrent ovarian cancer. Clin Transl Oncol 2009;11:753-9.

23. Cotte E, Glehen O, Mohamed F, et al. Cytoreductive surgery and intraperitoneal chemohyperthermia for chemoresistant and recurrent advanced epithelial ovarian cancer: prospective study of 81 patients. World J Surg 2007;31:1813-20.

24. Piso P, Dahlke MH, Loss M, et al. Cytoreductive surgery and hyperthermic intraperitoneal chemotherapy in peritoneal carcinomatosis from ovarian cancer. World J Surg Oncol 2004;2:21.

25. Cascales-Campos P, López-López V, Gil J, et al. Hyperthermic intraperitoneal chemotherapy with 
paclitaxel or cisplatin in patients with stage III-C/IV ovarian cancer. Is there any difference? Surg Oncol 2016;25:164-70.

26. Muñoz-Casares FC, Medina-Fernández FJ, ArjonaSánchez Á, et al. Peritonectomy procedures and HIPEC in the treatment of peritoneal carcinomatosis from ovarian cancer: Long-term outcomes and perspectives from a high-volume center. Eur J Surg Oncol 2016;42:224-33.

27. Di Giorgio A, De Iaco P, De Simone M, et al. Cytoreduction (peritonectomy procedures) combined with hyperthermic intraperitoneal chemotherapy (HIPEC) in advanced ovarian cancer: retrospective Italian multicenter observational study of 511 cases. Ann Surg Oncol 2017;24:914-22.

28. Foster JM, Sleightholm R, Smith L, et al. The American Society of Peritoneal Surface Malignancies MultiInstitution evaluation of 1,051 advanced ovarian cancer patients undergoing cytoreductive surgery and HIPEC: An introduction of the peritoneal surface disease severity score. J Surg Oncol 2016;114:779-84. Erratum in: J Surg Oncol. 2018 Sep;118(4):720. doi: 10.1002/jso.24603. Epub 2017 Feb 20.

29. Cascales-Campos PA, Gil J, Gil E, et al. Treatment of microscopic disease with hyperthermic intraoperative intraperitoneal chemotherapy after complete cytoreduction improves disease-free survival in patients with stage IIIC/ IV ovarian cancer. Ann Surg Oncol 2014;21:2383-9.

30. Gonzalez Bayon L, Steiner MA, Vasquez Jimenez W, et al. Cytoreductive surgery and hyperthermic intraperitoneal chemotherapy for the treatment of advanced epithelial ovarian carcinoma: upfront therapy, at first recurrence, or later?. Eur J Surg Oncol 2013;39:1109-15.

31. Parson EN, Lentz S, Russell G, et al. Outcomes after cytoreductive surgery and hyperthermic intraperitoneal chemotherapy for peritoneal surface dissemination from ovarian neoplasms. Am J Surg 2011;202:481-6.

32. Lim MC, Kang S, Choi J, et al. Hyperthermic intraperitoneal chemotherapy after extensive cytoreductive surgery in patients with primary advanced epithelial ovarian cancer: interim analysis of a phase II study. Ann Surg Oncol 2009;16:993-1000.

33. D'Hondt V, Goffin F, Roca L, et al. Interval cytoreductive surgery and hyperthermic intraperitoneal chemotherapy in first-line treatment for advanced ovarian carcinoma: a feasibility study. Int J Gynecol Cancer 2016;26:912-7.

34. Coccolini F, Campanati L, Catena F, et al. Hyperthermic intraperitoneal chemotherapy with cisplatin and paclitaxel in advanced ovarian cancer: a multicenter prospective observational study. J Gynecol Oncol 2015;26:54-61.

35. Deraco M, Kusamura S, Virzì S, et al. Cytoreductive surgery and hyperthermic intraperitoneal chemotherapy as upfront therapy for advanced epithelial ovarian cancer: multi-institutional phase-II trial. Gynecol Oncol 2011;122:215-20.

36. Sugarbaker PH, Technical handbook for the integration of cytoreductive surgery and perioperative intraperitoneal chemotherapy into the surgical management of gastrointestinal and gynecologic malignancy. Grand Rapids, Michigan. 2005:12-24.

37. Virzì S, Iusco DR, Bonomi S, et al. Hyperthermic Intraperitoneal Chemotherapy (HIPEC) Techniques. Treatment of Peritoneal Surface Malignancies: Springer, 2015:155-68.

38. Elias D, Antoun S, Goharin A, et al. Research on the best chemohyperthermia technique of treatment of peritoneal carcinomatosis after complete resection. Int J Surg Investig 2000;1:431-9.

39. Bristow RE, Karlan BY, Chi DS. Surgery for ovarian cancer: CRC Press, 2015.

40. González-Moreno S, González-Bayón L, OrtegaPérez G. Hyperthermic intraperitoneal chemotherapy: methodology and safety considerations. Surg Oncol Clin N Am 2012;21:543-57.

41. Jacquet P, Averbach A, Stephens AD, et al. Heated intraoperative intraperitoneal mitomycin $\mathrm{C}$ and early postoperative intraperitoneal 5 -fluorouracil: pharmacokinetic studies. Oncology 1998;55:130-8.

42. Stephens AD, Alderman R, Chang D, et al. Morbidity and mortality analysis of 200 treatments with cytoreductive surgery and hyperthermic intraoperative intraperitoneal chemotherapy using the coliseum technique. Ann Surg Oncol 1999;6:790-6.

43. Rodríguez Silva C, Moreno Ruiz FJ, Bellido Estévez I, et al. Are there intra-operative hemodynamic differences between the Coliseum and closed HIPEC techniques in the treatment of peritoneal metastasis? A retrospective cohort study. World J Surg Oncol 2017;15:51.

44. Halkia E, Tsochrinis A, Vassiliadou DT, et al. Peritoneal carcinomatosis: intraoperative parameters in open (coliseum) versus closed abdomen HIPEC. Int J Surg Oncol 2015;2015:610597.

45. Fujimura T, Yonemura Y, Fujita H, et al. Chemohyperthermic peritoneal perfusion for peritoneal dissemination in various intra-abdominal malignancies. Int Surg 1999;84:60-6.

46. Yonemura Y, Ninomiya I, Kaji M, et al. Prophylaxis with 
intraoperative chemohyperthermia against peritoneal recurrence of serosal invasion-positive gastric cancer. World J Surg 1995;19:450-4.

47. Lotti M, Capponi MG, Piazzalunga D, et al. Laparoscopic HIPEC: a bridge between open and closed-techniques. J Minim Access Surg 2016;12:86.

48. Näslund Andréasson S, Anundi H, Thorén SB, et al. Is Platinum Present in Blood and Urine from Treatment Givers during Hyperthermic Intraperitoneal Chemotherapy? J Oncol 2010;2010:649719.

Cite this article as: $\mathrm{Ha} \mathrm{HI,} \mathrm{Lim} \mathrm{MC.} \mathrm{How} \mathrm{do} \mathrm{we} \mathrm{perform}$ hyperthermic intraperitoneal chemotherapy in ovarian cancer? —a narrative review. Gland Surg 2021;10(3):1235-1243. doi: $10.21037 /$ gs-20-425
49. Schmid K, Boettcher M, Pelz J, et al. Investigations on safety of hyperthermic intraoperative intraperitoneal chemotherapy (HIPEC) with Mitomycin C. Eur J Surg Oncol 2006;32:1222-5.

50. Stuart OA, Stephens AD, Welch L, et al. Safety monitoring of the coliseum technique for heated intraoperative intraperitoneal chemotherapy with mitomycin C. Ann Surg Oncol 2002;9:186-91. 